\title{
Candy-like sign during endoscopic ultrasound- guided choledochoduodenostomy as an indication of the long distance between the bile duct and duodenal wall
}

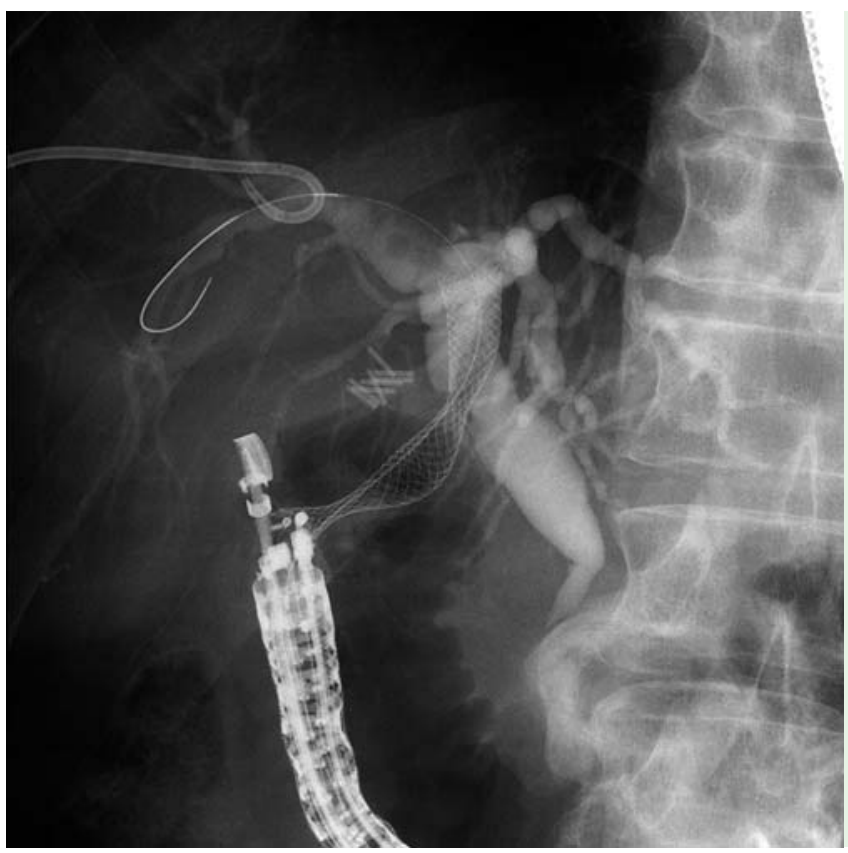

Fig. 1 Radiographic image showing the candy-like sign of a partially covered selfexpandable metallic stent resulting from a large gap between the common bile duct and the duodenal wall during endoscopic ultrasound-guided choledochoduodenostomy.

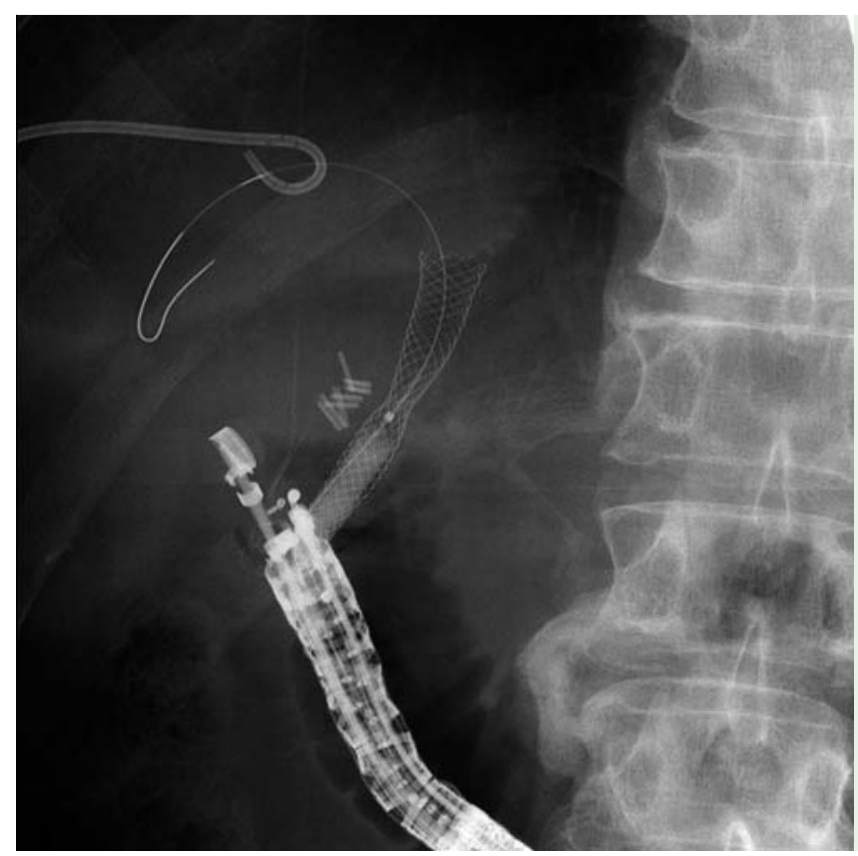

Fig. 3 Radiograph showing balloon dilation of the first partially covered self-expandable metallic stent.

Endoscopic ultrasound-guided choledocoduodenostomy (EUS-CDS) using a covered self-expandable metallic stent (SEMS) is an established alternative drainage technique for patients in whom endoscopic retrograde cholangiopancreatography has failed $[1,2]$. This report describes the case of a patient who underwent successful EUS-CDS with a partially covered SEMS placed far from the duodenal wall.

A 66-year-old man with locally advanced pancreatic head cancer was admitted to our hospital. He had undergone percutaneous transhepatic biliary drainage

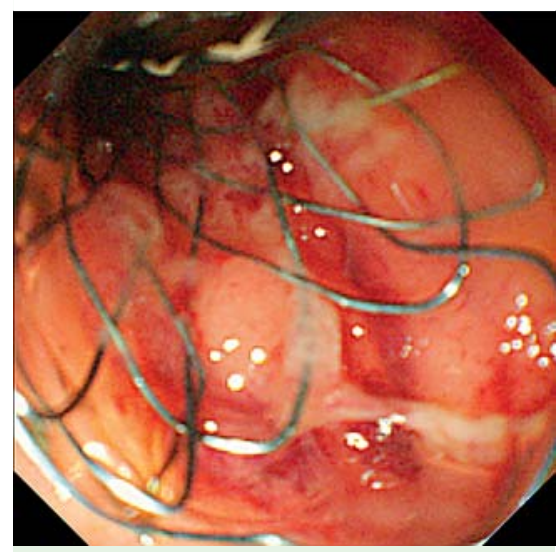

Fig. 2 Endoscopic image showing migration of the distal end of the partially covered selfexpandable metallic stent during endoscopic ultrasound-guided choledochoduodenostomy.

(PTBD) at another hospital 1 week earlier because of failed selective bile duct cannulation. He experienced right flank pain after PTBD. As the patient wanted the PTBD tube removed, we opted to perform EUS-CDS rather than antegrade stenting. After puncture of the common bile duct from the first part of the duodenum using a 19-gauge needle (Echo Tip Ultra; Cook Japan, Tokyo, Japan), a 0.025-inch guidewire (VisiGlide; Olympus Medical Systems Corp., Tokyo, Japan) was inserted into the intrahepatic bile duct. Fistula dilation was then performed using a $6-\mathrm{Fr}$ wire-guided diathermic dilator (CystoGastro-Set; Endo-Flex Gmbh, Voerde, Germany) with a blended cut mode. Insertion of a partially covered SEMS (WallFlex, 10× $60 \mathrm{~mm}$; Boston Scientific Japan, Tokyo, Japan) was then attempted through the fistula. The EUS-CDS procedure was performed quickly; however, the SEMS revealed a candy-like sign in the form of a large gap between the bile duct and duodenum, and this sign warrants caution as it indicates distal migration and bile leakage ( $\bullet$ Fig. 1 and $\bullet$ Fig. 2; $\bullet$ Video 1 ).

We therefore attempted additional stenting using a fully covered SEMS (Bonastent, $10 \times 60 \mathrm{~mm}$; Standard Sci Tech, Seoul, Korea), but this stent could not be passed through the first partially covered stent. Balloon dilation (Hurricane RX Biliary Bal- 


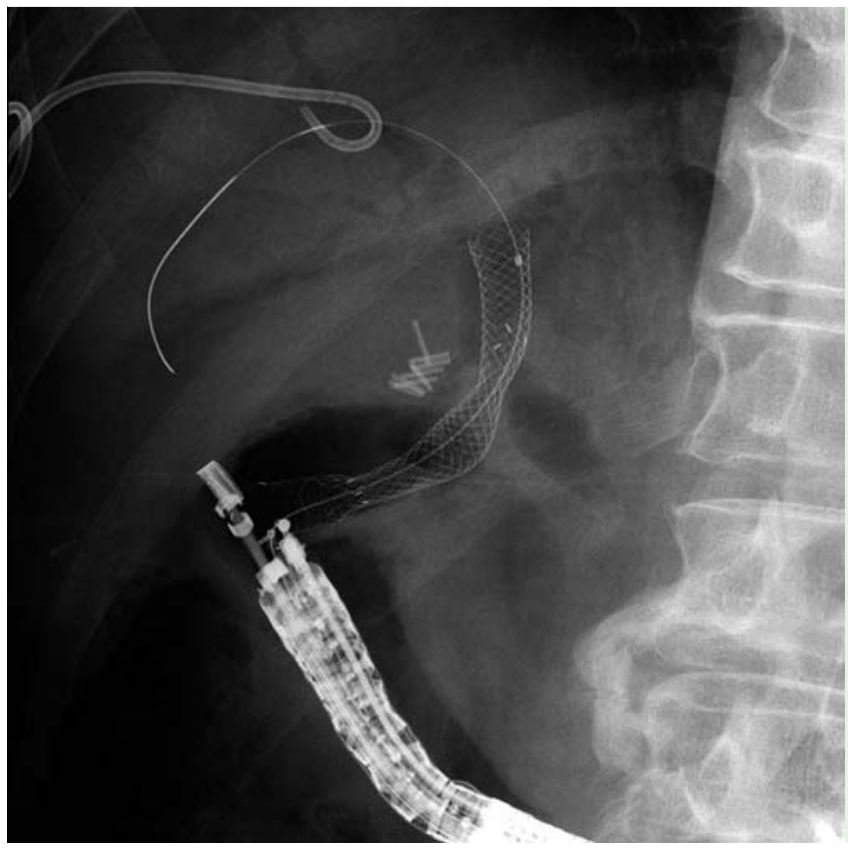

Fig. 4 Radiograph showing the second fully covered self-expandable metallic stent (SEMS) placed across the first partially covered SEMS.

loon Dilation Catheter; Boston Scientific Japan) of the first partially covered SEMS was performed ( Fig.3; Video 1). Finally, a second fully covered SEMS was placed across the fistula through the first partially covered stent without any complication ( Fig.4 and $\bullet$ Fig. 5; $\bullet$ Video 1 ). We were successful in saving this patient using placement of an additional fully covered SEMS. Caution should be taken in the event of the rare and dangerous candy-like sign, which indicates a long distance between the bile duct and duodenum, during EUS-CDS with covered SEMS placement.

\section{Video 1}

Endoscopic rescue technique for gap formation of the partially covered self-expandable metallic stent (SEMS), which was dilated using a balloon catheter followed by placement of a fully covered SEMS.
Endoscopy_UCTN_Code_CPL_1AL_2AD

\section{Competing interests: None}

\section{Hiroshi Kawakami, Masaki Kuwatani, Kazumichi Kawakubo, Taiki Kudo, Yoko Abe, Kimitoshi Kubo, Yoshimasa Kubota, Naoya Sakamoto}

Department of Gastroenterology and Hepatology, Hokkaido University Graduate School of Medicine, Sapporo, Japan

\section{References}

1 Itoi $T$, Isayama $H$, Sofuni $A$ et al. Stent selection and tips on placement technique of EUS-guided biliary drainage: transduodenal and transgastric stenting. J Hepatobiliary Pancreat Sci 2011; 18: 664-672

2 Kawakubo K, Isayama $\mathrm{H}$, Kato $\mathrm{H}$ et al. A multicenter retrospective study of endoscopic ultrasound-guided biliary drainage (EUSBD) for malignant biliary obstruction in Japan. J Hepatobiliary Pancreat Sci 2014; 21: $328-334$

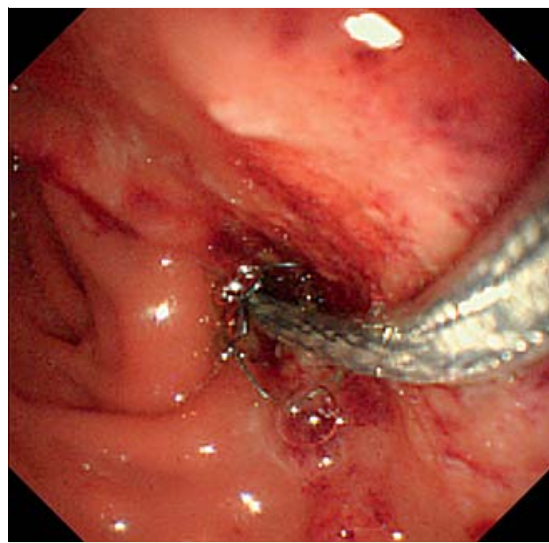

Fig.5 Endoscopic image showing the second fully covered self-expandable metallic stent (SEMS) advanced over a guidewire through the first partially covered SEMS.

Bibliography

DOI http://dx.doi.org/

10.1055/s-0034-1377390

Endoscopy 2014; 46: E406-E407

(c) Georg Thieme Verlag KG

Stuttgart · New York

ISSN 0013-726X

Corresponding author

\section{Hiroshi Kawakami, MD, PhD}

Department of Gastroenterology and Hepatology Hokkaido University Graduate School of Medicine Kita 15, Nishi 7

Kita-ku

Sapporo 060-8638

Japan

Fax: +81-11-7067867

hiropon@med.hokudai.ac.jp 